\title{
Noise Cancellation Employing Adaptive Digital Filters for Mobile Applications
}

\author{
A.M. Prasanna Kumar ${ }^{1}$, S.M. Vijaya ${ }^{2}$ \\ ${ }^{1}$ Department of Electronics \& Communication Engineering, ACS College of Engineering, Bengaluru, Karanataka, India. \\ ${ }^{2}$ Department of Electronics \& Communication Engineering, Rajarajeswari College of Engineering, Bengaluru,
} Karanataka, India.

\begin{tabular}{l}
\hline \hline Article Info \\
\hline Article history: \\
Received Mar 27, 2019 \\
Revised Mar 11, 2020 \\
Accepted Mar 13, 2020 \\
\hline
\end{tabular}

\section{Keywords:}

Adaptive Filter, Noise Cancellation, Convergence Complexity, Bit Error Rate, Adaptive Noise Cancellation

\begin{abstract}
The persistent improvement of the hybrid adaptive algorithms and the swift growth of signal processing chip enhanced the performance of signal processing technique exalted mobile telecommunication systems. The proposed Artificial Neural Network Hybrid Back Propagation Adaptive Algorithm (ANNHBPAA) for mobile applications exploits relationship among the pure speech signal and noise corrupted signal in order to estimate of the noise. Linear networks are gets adjusted at each time step based over new input and target vectors find weights and biases that reduces the networks sum squared error for recent input and target vectors. Networks of this kind are quite oftenly used for error cancellation, speech signal processing and control systems. Noise in an audio signal has become major problem and hence mobile communication systems are demanding noise-free signal. In order to achieve noise-free signal various research communities have provided significant techniques. Adaptive noise cancellation (ANC) is a kind of technique which helps in estimation of un-wanted signal and removes them from corrupted signal. This paper introduces an Adaptive Filter Based Noise Cancellation System (AFNCS) that incorporates a hybrid back propagation learning for the adaptive noise cancellation in mobile applications. An extensive study has been made to explore the effects of different parameters, such as number of samples, number of filter coefficients, step size and noise level at the input on the performance of the adaptive noise cancelling system. The proposed hybrid algorithm consists all the significant features of Gradient Adaptive Lattice (GAL) and Least Mean Square (LMS) algorithms. The performance analysis of the method is performed by considering convergence complexity and bit error rate (BER) parameters along with performance analyzed with varying some parameters such as number of filter coefficients, step size, number of samples and input noise level. The outcomes suggest the errors are reduced significantly when the numbers of epochs are increased. Also incorporation of less hidden layers resulted in negligible computational delay along with effective utilization of memory. All the results have been obtained using computer simulations built on MATLAB platform
\end{abstract}

Copyright (C) 2019 Institute of Advanced Engineering and Science. All rights reserved.

\footnotetext{
Corresponding Author:

Dr.A.M. Prasanna Kumar,

Professor,Department of Electronics \& Communication Engineering

ACS College of Engineering, Bengaluru, Karanataka, India

E-mail: amprasannakumar@acsce.edu.in
}

\section{INTRODUCTION}

In wired or wireless communication systems, noise cancellation has become a prime concern and considered an open research problem in current era of mass communication of data all over the world. The mechanism and technologies are invented in recent past for noise cancellation from the noise-containing desired signal. The existence of noise in the desired signal may distort the received signal with random manner 
which may have several sources. From the researches of Qadri et al. [1] and Riahi Manesh et al. [2] found that some of the sources like a) non-linearity exist at RF frontend b) existence of time-varying thermal noise at receiver end and c) noise interference from adjacent environment. In addition several other factors are affecting the received signal as cross talk and electromagnetic interference. Over the few decades, several denoising techniques are addressed in Qadri et al. [3], Tandra et al. [4] and Zeng et al. [5] and are divided as gradientdescent adaptive filter algorithm (AFA) and non-gradient AFA. The Gradient descent is a steepest descent which is a multivariate optimization approach and is initialized by assigning initial value for a negative gradient to achieve desired local minimum. Earlier various algorithms were provided to identify this desired signal. The LMS algorithm considered as significant with respect to need of computational efficiency and storage ability at low convergence speed. Also normalized LMS algorithm was considered at moderate sped of convergence in turn its response is sluggish for colored input signals.

Further RLS algorithm was considered an effect with respect to high speed of convergence and tracking ability. However, RLS yields high computational cost. This paper aims to design an Artificial Neural Network (ANN) based on hybrid back propagation algorithm to achieve better noise cancellation through adaptive control. Noise-reduction-ratio is the ratio of noise power to the error power. This paper categorized as: section 1 on introduction, section 2-related work, and section 3 gives the proposed solution, section 4 relates the algorithm implementation and section 5 presents the discussion of obtained results, while section 6 conveys the conclusion of the paper.

\section{RELATED WORK}

The prior section involves with the discussion of the most recent researches subjected with the mobile applications with different techniques adopted to offer better performance by considering BER, SNR, noise cancellation, interference cancellation, correlation, computational complexity as performance parameters. Recently the exponential growth of the mobile applications usage is creating huge traffic in wireless broadband technologies. In Alom and Li [2010] [6], comparative analysis of beam forming algorithm is performed with respect to correlation values at different angle of signal arrival by using back propagation algorithm. The work towards achieving accurate results for noise cancellation, echo cancellation and equalization is found in Kayode et al. (2014) [7] that implement the LMS based adaptive filtering technique for digital audio signals. The [7] simulation result come up with accurate and desired input/output signal by noise signal removal.

Similarly, an experimentation work is observed in Mohammed (2014) [8] that adopt the adaptive noise cancellation for health monitoring applications. Here, adaptive filters and modified LMS algorithms were used to eliminate the interference and noise from the mobile phones respectively. A work considering the mobile traffic issue is found in Yoon et al. (2014) [9] and have offered a multicast resource allocation scheme for 4G networks. The experimental analysis of [9] is incorporated with the modulation and coding scheme by considering average PSNR as performance parameter with significant improvement than existing schemes. The implementation of Error Back Propagation (EBP) is found in Roy and Rodrigues (2014) [10] for noise cancellation in the echo corrupted signal by building correlation with pure signal. Here, the voice/speech data are trained using ANN and analyzed [10] performance with respect to SNR, echo tracking, error tracking, echo variance from the recovered pure signal. Most of the researches were incorporated with an aim of interference cancellation schemes for the mobile communication and are perform well under synchronous environment which are not been handled with GMSK modulation schemes. The solution to this scenario is presented in Ruder et al. (2015) [11] with proper modifications in the conventional GSM systems towards the effects of asynchronous co-channel interferences and complexity. The outcome of the modified GSM system [11] gives the robust results against such asynchronous interferences than unmodified GSM systems.

A significant research towards the phase noise cancellation in shortest range of communications is observed in Zhang et al. (2016) [12] that correlate the quantization of noise cancellation with Least Mean Square (LMS) algorithms yielding high immunity and linearity. This [12] approach has achieved area optimization as well as power optimization with high data rates. Towards the noise removal for real-time sinusoidal signals in healthcare application Kelly et al. (2016) [13] have offered an adaptive filter approach which tracks the sinusoidal frequency and achieved narrow bandwidth results. The experimentation was done with Electro-Cortico-Graphic (ECoG) neural data consisting of power line noise and outcomes with enhanced SNR. Similarly, Chilipi et al. (2016) [14] worked for distributed power generation system through adaptive filters for noise/harmonics cancellations to achieve power quality enhancement.

Further, Garcia et al. (2016) [15] used adaptive filter to have noise suppression for echo cancellation, channel equalization, array beam forming in surveillance, tracking and target localization applications. A review work on noise cancellation using adaptive filter based LMS algorithm is presented in Dixit and Nagaria (2017) [16] by considering computational complexity and convergence rate. The similar direction of research is performed by Zheng et al. (2017) [17] and introduced the robust approach by utilizing the adaptive filtering 
algorithms for acoustic echo cancellations.The[17] approach outcomes with performance improvement with respect to sparsity of impulse response as well as differentiable cost function.

Similarly, Zhang et al. (2017) [18] have come up with a robust solution for echo cancellation application by using LMS algorithms. The outcome of the simulation using Monte Carlo suggests that the proposed [18] gives robustness in different environments. With an intention to suppression of residual self-interference, Ahmed and Tsimenidis (2018) [19] have used adaptive mean squared error filter for iterative decoding/detective. Here, both the Rayleigh fading and AWGN channels were considered for modulation and its simulation outcomes high light the system performance with respect to BER, SNR as performance parameters. A novel implementation of adaptive filtering algorithm is found in Menguc (2018) [20] for quaternion-valued least-mean kurtosis (QLMK) method. This [20] method is significant with applicability for wide range of noise signals cancellation and improvement in convergence, steady-state error. On analyzing the existing researches it is observed that very rare works are incorporated with adaptive approach for noise cancellation. Among these, most of the works adaptive approaches are implemented only the LMS algorithms. Most of the researches were used back propagation or feed forward propagation approach for noise cancellation and minimize noise error rate. This paper introduces an artificial neural network based adaptive approach which incorporates hybrid back propagation for noise cancellation by using different algorithms like LMS, Gradient adaptive algorithm and hybrid adaptive algorithm. The proposed Adaptive Filter Based Noise Cancellation System (AFNCS) considers the convergence error and computational complexity for performance analysis.

\section{PROPOSED SOLUTION}

In voice communication systems, noise cancellation using adaptive digital filter is a renowned technique for extracting desired speech signal by eliminating noise from the speech signal corrupted by noise.For noise cancellation various gradient adaptive lattice (GAL) and LMS algorithms used. Recently, the hybrid adaptive algorithms with neural networks have gained popularity in cancelling the noise available in communication system. The working principle of the proposed intelligent adaptive filter based noise cancellation system (AFNCS) is the continuation of prior work (Kumar et al. [21]) which is further empirically designed and simulated to enhance the performance of the input synthetic signal with respect to noise cancellation. In this, a hybrid back propagation algorithm is introduced by which learning of multi-layer network is achieved. The noise analysis of the system is performed by using artificial neural network (ANN).

This intelligent hybrid back propagation algorithm involves both GAL and LMS algorithm. The prime objective of the proposed intelligent AFNCS is to acquire signal from reference signal and output noisy signal, among these signal noise is eliminated by subtracting the reference signal and noisy signal with original signal. The use of AFNCS can significantly restore the original signal by eliminating the noise by using adaptive control and adjustment of weights through ANN. The following Figure.1, indicates the block representation of the AFNCS which intakes the input signal " $\mathrm{i}(\mathrm{t})$ " and generates output signal "O(t)" by using adaptive system and reference signal " $\mathrm{R}(\mathrm{t})$ ". Finaly, the error signal $\mathrm{e}(\mathrm{t})$ is computed by finding the difference among reference signal and output signal (as given in Eq. (1)).

$$
e(t)=R(t)-O(t)
$$

\section{Where ' $t$ ' represents number of iterations}

The adoption of hybrid algorithm considers this error signal e(t) to generate a function for execution. This function performs the computation of desired filter coefficients. The minimized error rate indicates that output signal is same similar as that of original signal. Here back propagation algorithms are used to evaluate the error rate of each neuron. The following Figure. 2 highlights the structural model of back propagation layer diagram of ANN network. The layer diagram of ANN network is made up of three layers comprising input layer, hidden layer and output layer. The hidden layer existing in between input layer and output layer which couples both the layers. The overall back propagation network is affected by one neuron error. The network allows audio or speech signal to propagate via ANN and provides output signal. As given in Eq. (1) the error results of the output layer are computed and this error is forwarded back to input layer through hidden layer until the desired output is obtained.

Further, to minimize its error signal, adjustment of weight is performed for each neurons. The proposed hybrid algorithm combines both the back propagation algorithm of LMS and GAL which helps to tackle slow convergence.

IJEEI, Vol. 8, No. 1, March 2020 : 112-122 


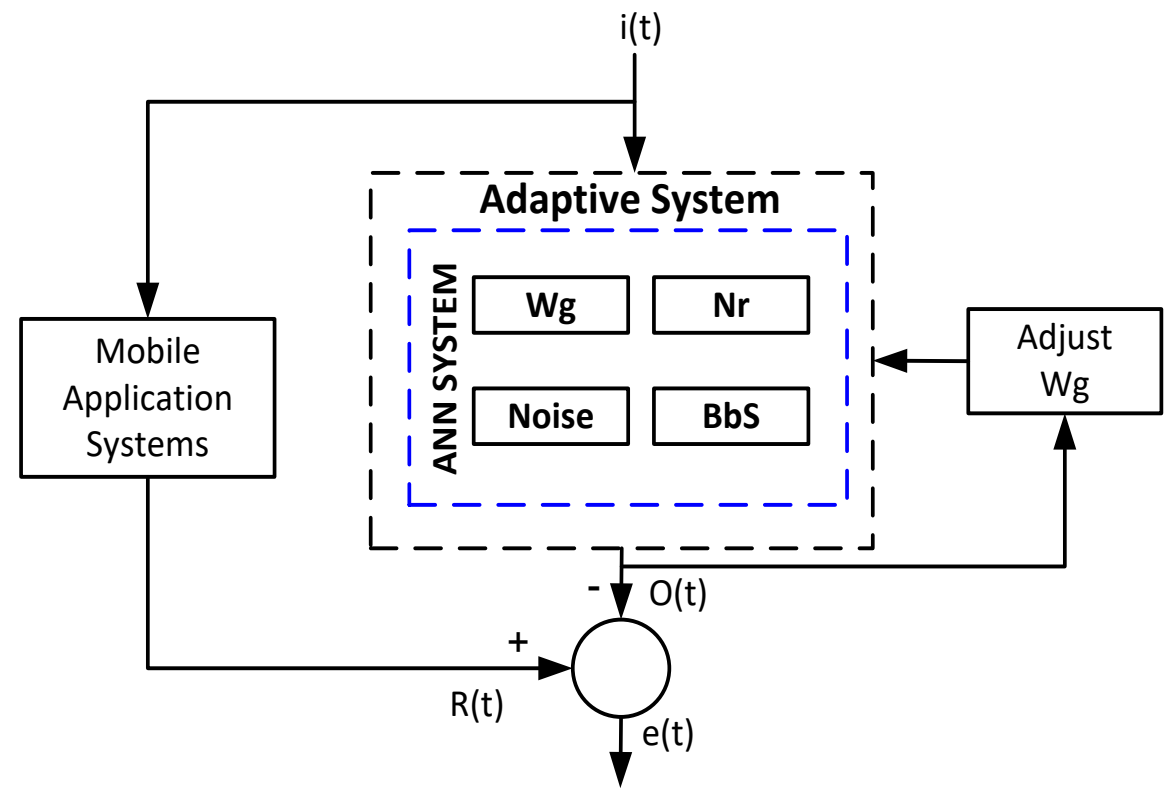

Figure 1. Proposed Adaptive Filter Based Noise Cancellation System (AFNCS)

The proposed AFNCS adopts adaptive filtering for implementation of ANN and also adopts a control system for adjustment of adaptive filtering parameters. The elements connection is trained with ANN by weight adjustment. The output of ANN can be obtained by using below formula (as given in Eq. (2)). The following table 1 , indicates the parameters used in design.

$$
A N N_{\text {out }}=\sum i(t) \times W_{g}
$$

Each inputs are accompanied by a weight.

If, $\sum W_{g} \geq T h$

then the output of ANN will be 1 given in Eq. (3)

$$
A N N_{\text {out }}=1
$$

Table 1. Parameters used in intelligent AFNCS system

\begin{tabular}{ll} 
Parameter & Description \\
\hline $\mathrm{i}(\mathrm{t})$ & Signal applied at input \\
$\mathrm{R}(\mathrm{t})$ & Reference Signal \\
$\mathrm{O}(\mathrm{t})$ & Signal obtained at output \\
$\mathrm{e}(\mathrm{t})$ & Error Signal \\
$\mathrm{Wg}$ & Weight \\
$\mathrm{Nr}$ & Neurons \\
$\mathrm{Bps}$ & Baseband Signals \\
$\mathrm{Th}$ & Threshold $(0$ to 1$)$ \\
$\mathrm{ANN}$ out & Output of ANN \\
\hline
\end{tabular}

However, if $\sum W_{g}<T h$, then the output of ANN will be 0

(given in Eq. (4))

$$
A N N_{\text {out }}=0
$$

To get the desired output signal ANN needs to be adjusted for the weights with respect to input samples. The formation of the ANN system (shown in Figure.2) is done by considering three layers such as the output layer, hidden layers and the input layer. The audio signal is fed to the input layers having neurons. The hidden layer features in minimizing the reducing the error rate to achieve the respective output. The output layer does the competition of neuron nodes based on the requirement of output. 


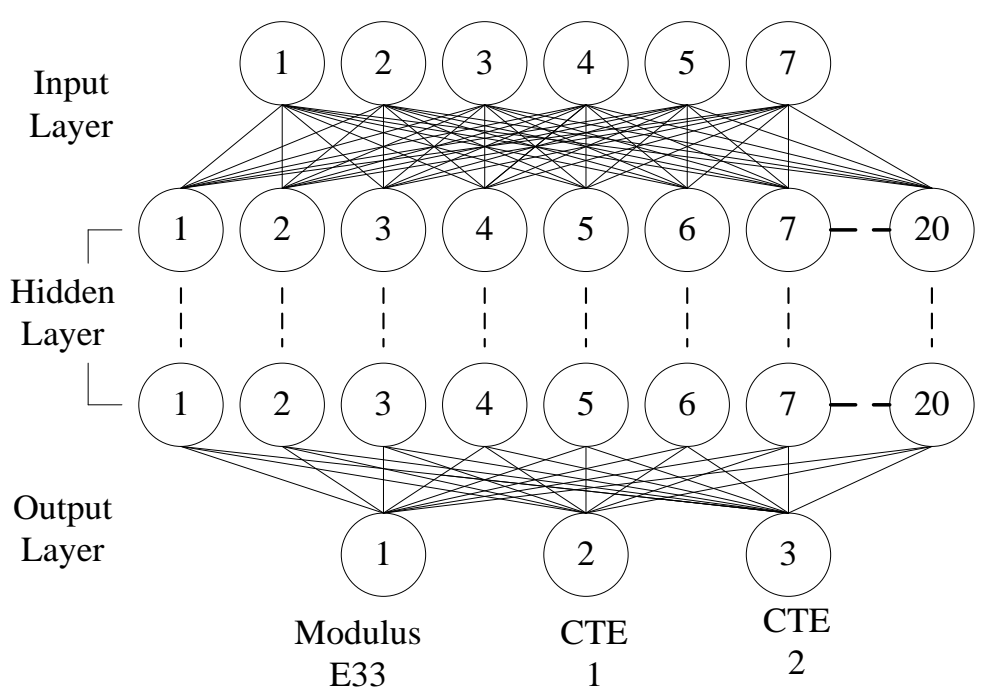

Figure 2. Layer diagram of ANN

In the proposed ANFCS implements the LMS mechanism to compute the instantaneous value of the gradient vector. Then, the minimization of the MSE is performed by varying the filter weights. For each iteration of the adaptive filter, the optimized Weiner solution is obtained which can be referenced with Eq. (5) where $n, S_{t}$, Ws(n), I(n) represents the time, step size, adaptive filter coefficient and input vector respectively.

$$
W s(n+1)=W s(n)+\left(S_{t} \times e(n) \times I(n)\right.
$$

In order to avoid the instability, output divergence and convergence time, the optimal value of $\mathrm{S}_{\mathrm{t}}$ is selected. Further, to reduce the error, negative gradient functions are considered.

The following Eq. (6) and (7) gives the computation of e(n) and I(n) respectively.

$$
\begin{aligned}
& e(n)=R(n)-\left(W_{g}(n) \times I(n)\right) \\
& I(n)=\left[i(n) \times i(n-1) \ldots x(n-)-\left(W_{g}(n) \times i(n+1)^{T}\right)\right]
\end{aligned}
$$

Then the LMS Algorithm can be described as

$$
\begin{aligned}
& O(n)=\sum_{i=0}^{M-1} W i(n) \times i(n-i) \\
& e(n)=R(n)-O(n) \\
& W i(n+1)=w_{i}(n)+\left(\operatorname{St}(n)^{*} \times i(n-i)\right) \\
& O(n)=w_{0}(n)^{*} \times i(n)
\end{aligned}
$$

Where, $W(n)=\left[w(n) \times w_{1}(n) \ldots .\left(W_{l L-1}(n)^{T}\right.\right.$ is a coefficient vector

In the proposed method, the convergence rate of error signal increases with the value of $\mathrm{S}_{\mathrm{t}}$. LMS mechanism is adopted in the proposed method because of its easier implementation, simple computational, dynamic usage of memory ability and is performed by adjusting filter coefficient for error minimization.

\section{ALGORITHMS}

In a numerical computing environment proposed AFNCS is modeled by means of soft computation based algorithm design and implementation. The system specifications which are required to know the AFNCS algorithm performance includes a 64-bit operating system, an x64-based processor supported with 4.00 GB installed memory (RAM), where the processor type is Intel ${ }^{\circledR}$ Core $^{\mathrm{TM}} \mathrm{i}-8250 \mathrm{U}, \mathrm{CPU} @ 1.60 \mathrm{GHz}, 1.80 \mathrm{GHz}$.The following algorithm exhibits the steps associated with AFNCS design goals to achieve cost-effective adaptive noise cancellation from a sinusoidal (speech signal). 
Input: $\mathrm{i}(\mathrm{t}), \mathrm{R}(\mathrm{t})$

Output: e (t), O (t)

1. START

2. Initialize $\mathrm{w}_{\mathrm{g}}, \mathrm{N}_{\mathrm{r}}, \mathrm{B}_{\mathrm{b}} \mathrm{S}, \mathrm{i}(\mathrm{t}), \mathrm{R}(\mathrm{t})$ where $\mathrm{t} \in \mathrm{Z}^{+}$which is iteration number

3. Initialize hybrid adaptive Filter Configuration with LMS and GAL

4. $f$ for $(i=1: t)$

5. Enable ANN

6. Compute $\mathrm{O}(\mathrm{t})=f_{\mathrm{AFNCS}}(\mathrm{i}(\mathrm{t}))$

7. Compare $\mathrm{O}(\mathrm{t})$ with $\mathrm{R}(\mathrm{t})$

8. $\mathrm{e}(\mathrm{t})=\mathrm{R}(\mathrm{t})-\mathrm{O}(\mathrm{t})$

9. Training Input $\left[\mathrm{a}_{\mathrm{ij}}\right]_{12 \times 4}$, Training ${ }_{\text {Output }}\left[\mathrm{b}_{\mathrm{ij}}\right]_{12 \times 1}$

10. Initialize hidden neurons, epochs

11. Validate the number of rows equality among input data and output data vector, if mismatch error.

12. Compute $\mu_{\text {mean }}=\frac{1}{n} \sum_{i=1, j=1}^{n}[$ aij] $12 \mathrm{x} 4$ where training vector is made up of $\mathrm{n}$ scalar observations

13. Compute $\sigma_{\text {ip }}=$ standard deviation of SNR from

AFNCS at different dB's.

14. start Learning

15. for (iteration $=1$ : epochs)

16. Compute learning rate

17. Adjust weight hidden $\rightarrow$ Output

18. Adjust weight input $\rightarrow$ hidden

19. Normalize inputs and output vectors from (0 to 1$)$ using ANN

20. Compute error $\mathrm{e}(\mathrm{t})=\mathrm{R}(\mathrm{t})-\mathrm{O}(\mathrm{t})$

21. end

22. end

23. END

The above algorithm represents the computational steps associated with the proposed AFNCS which combines the strength and significant features of adaptive algorithms such GAL and LMS for the purpose of accomplishing multi-layer perceptron network. The proposed system incorporates a hybrid back propagation learning for the adaptive noise cancellation in mobile applications. The proposed hybrid algorithm consists of all the significant features of LMS and GAL algorithms. The above algorithm clearly shows that how incorporating ANN based adaptive learning AFNCS significantly reduces the error rate of an input speech signal from a soft-computing viewpoint. It also applies weight-adjustment in ANN which influences lower convergence performance with less iterative steps driven by non-recursive functions. The algorithm is designed simulated in a numeral computing platform, and the performance of the proposed AFNCS has been justified with respect to two different parameters such as processing time(s) and bit error rate (BER) from both complexity and signal quality viewpoint.

\section{RESULTS}

This section talks about the experimental outcome attained subsequently simulating the projected AFNCS in a numerical computing environment. Noise reduction ratio (in $\mathrm{dB}$ ). The proposed AFNCS considers training input of a vector [aij]12x 3 which is SNR values from different adaptive filters and further it assessed the above mentioned AFNCS algorithm which further results in Training output $=[$ aij] $12 \times 1$, which is a predicted/estimated SNR values for different signals of the proposed method at different dBs. 
Table 2. Comparative Performance Analysis

\begin{tabular}{llccr}
\hline \multicolumn{1}{c}{ At 30dB } & RLS[22] & FTF & GAL & AFNCS \\
\hline Chirp & 13.9296 & 12.0000 & 14.0000 & 9.7590 \\
Sinusoidal & 14.5297 & 14.9000 & 13.4000 & 7.5829 \\
Saw tooth & 12.7979 & 12.8500 & 12.1000 & 8.5157 \\
Audio & 13.0794 & 13.7000 & 12.8000 & 7.2004 \\
\hline At 10dB & RLS & FTF & GAL & AFNCS \\
\hline Chirp & 24.7287 & 24.9000 & 24.7000 & 9.4650 \\
Sinusoidal & 14.5130 & 14.6000 & 14.4000 & 8.1602 \\
Saw tooth & 12.7880 & 12.8000 & 12.7000 & 9.5066 \\
Audio & 25.5130 & 25.6000 & 25.5000 & 10.2760 \\
\hline & & & & \\
\hline At -10dB & RLS & FTF & GAL & AFNCS \\
\hline Chirp & 68.7400 & 68.8000 & 68.7000 & 21.4568 \\
Sinusoidal & 72.7454 & 72.8000 & 72.7000 & 23.3650 \\
Saw tooth & 71.4740 & 71.5000 & 71.4000 & 21.6489 \\
Audio & 46.6549 & 46.7000 & 46.6000 & 20.4271 \\
\hline
\end{tabular}

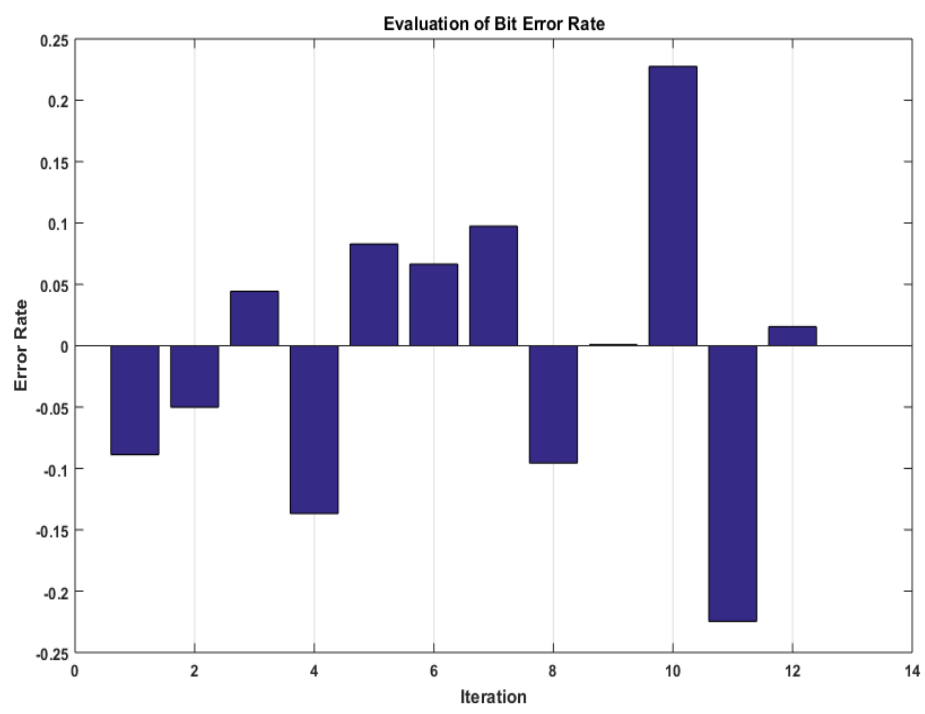

Figure 3. Error rate versus Number of Iterations before assessing with AFNCS

The following figure 3 and figure 4 exhibits the percentage of total error obtained from the output error signal, where the observation carried out shows that error has been reduced to a significant extent while the number of epochs increased to 5000 iterations.

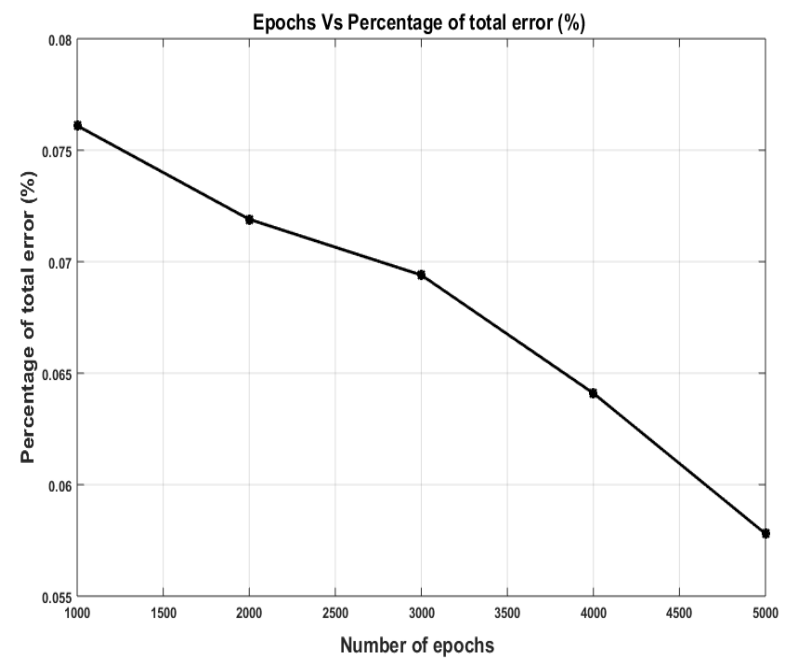

Figure 4. Evaluation of BER computation after assessing AFNCS 
It is also closely observed during the numerical computation that, number of hidden layers which having a relationship with computational delay. As the number of hidden layer increases delay will increase less the amount of hidden layer less will be computational overhead, which will result in the faster response. It will also lead to the effective utilization of system memory. The following Comparison Table- 2 shows a comparative performance analysis outcome of Noise Reduction Ratio from simulating the proposed AFNCS has been compared with RLS, FTF and GAL algorithms with respect to error [1].The comparative performance analysis considered three different types of the signal of different SNR values such as $30 \mathrm{~dB}, 10 \mathrm{~dB}$ and $-10 \mathrm{~dB}$.

A closer glance into the above table 2 indicates that the suggested AFNCS accomplishes better outcome as it yields very less output signal error as compared to the predictable baselines. It is also observed that incorporation of less hidden layers resulted in negligible computational delay along with effective utilization of memory. Along with performance evaluation of adaptive noise elimination employing NLMS algorithm is carried out. The operation is analyzed by changing some factors like number of filter coefficients, input noise level, step size and number of samples. All the results attained using computer simulations built on MATLAB platform. A recorded speech signal shown in Fig.5 with following characteristics has been taken into consideration to study the system performance.

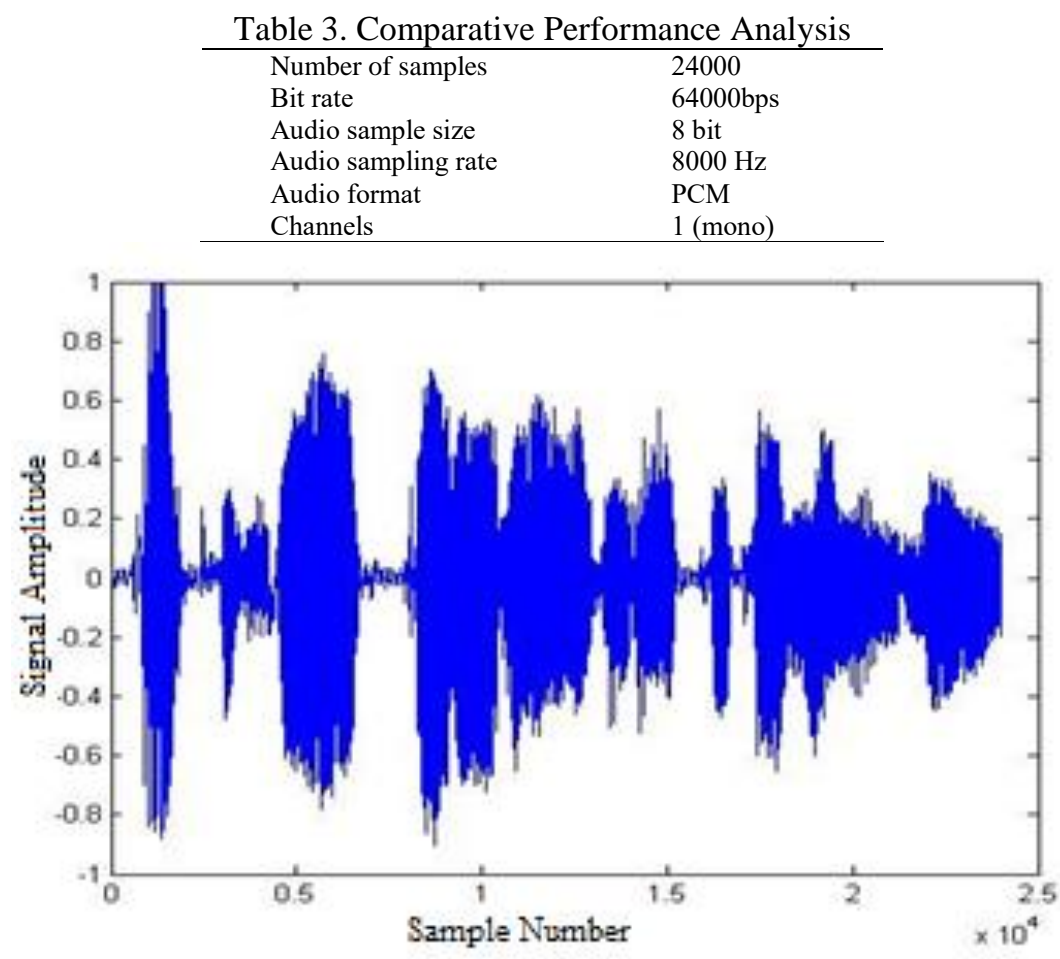

Figure 5. A speech signal

\subsection{Effects of number of filter coefficients}

Ten observations were made shown in table 4 [23] to evaluate the variations of the system performance with the number of filter coefficients. The system performance is measured by calculating noise reduction ratio (in $\mathrm{dB})$.

\subsection{Effects of step size}

The effects of step size (adaptive algorithm parameter) on the performance of the system are evaluated. The step size is increased from 0.01 to 0.02 and the system performance corresponding to respective step size is measured in terms of Noise Reduction Ratio (NRR). The simulation parameters and the results obtained for 20000 samples are tabulated in Table 4. [23].A graphical depiction of tabular data is shown in Fig.6. It is observed from tabulated values in Table 5. Plotted in Fig.7 that above a particular value of step size (0.03), the NRR gradually declines with increasing step size. Below that value, NRR gradually increases with the increase in step size. The optimum step size (at which the best noise reduction is seen) is 0.03 for the given simulation parameters. 
Table 4. Effects of number of filter coefficients [23]

Simulation parameters

Total number of samples: 20000

Noise power: $-10.00 \mathrm{~dB}$

No. of filter coefficients: 18

Step size: 0.01

Frequency range of colored noise: $1200 \mathrm{~Hz}-2000 \mathrm{~Hz}$

\begin{tabular}{cc}
\hline Number of filter coefficients & Noise Reduction Ratio (in dB) \\
\hline 3 & 27.0656 \\
4 & 28.7159 \\
5 & 28.9636 \\
6 & 29.1950 \\
7 & 29.9992 \\
10 & 29.1705 \\
12 & 29.4239 \\
14 & 29.1195 \\
16 & 29.4878 \\
18 & 28.6723 \\
\hline
\end{tabular}

Noise reduction ratio versus filter coefficient

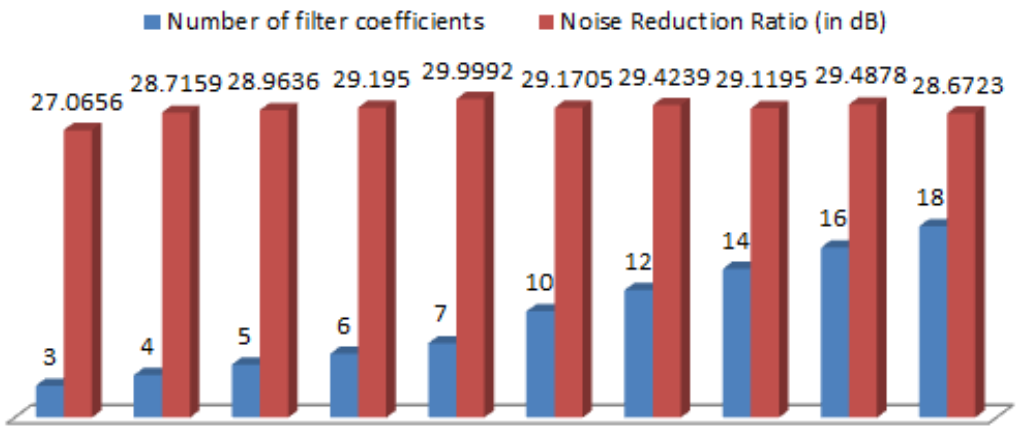

Figure 6. Noise reduction ratio versus filter coefficient

Table 5. Effects of step size [23]

Simulation parameters

Total number of samples: 15000

Noise power: $-10.0000 \mathrm{~dB}$

No. of filter coefficients: 18

Step size: 0.01

Frequency range of colored noise: $1200 \mathrm{~Hz}-2000 \mathrm{~Hz}$

\begin{tabular}{cc}
\hline Step Size & Noise Reduction Ratio (in dB) \\
\hline 0.01 & 26.2926 \\
0.02 & 26.6716 \\
0.03 & 27.1593 \\
0.04 & 26.9859 \\
0.05 & 26.0503 \\
0.06 & 25.4877 \\
0.07 & 24.9614 \\
0.08 & 24.8289 \\
0.09 & 23.408 \\
\hline
\end{tabular}

= Step Size $\quad$ Noise Reduction Ratio (in dB)

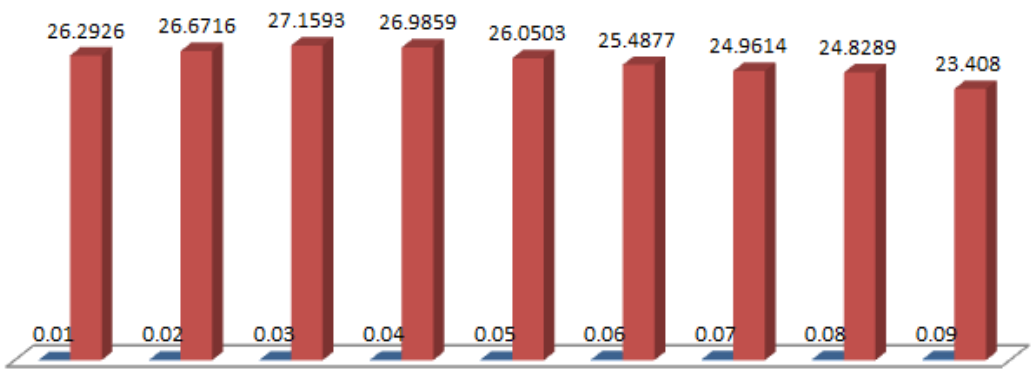

Figure 7. Variations of noise reduction ratio with step size 


\section{CONCLUSION}

This paper introduces an intelligent adaptive filter based noise cancellation system (AFNCS) by using LMS and GAL algorithms. The AFNCS is modelled in the numerical computing environment by means of soft computation based algorithm design and implementation. The performance of the proposed AFNCS is performed by considering convergence complexity and bit error rate as a performance parameter. From the analysis of the outcomes it is found that the errors are reduced significantly when the number of epochs is increased. From computational numerical data, it is found that the number of the hidden layers increases delay will increase and have a faster response the number of hidden layers should be less. The performance analysis of AFNCS considers chirp, sinusoidal, saw tooth and audio signals at an SNR value of 30,10 and -10db and are compared with Ferdouse et al. [22]. From, the comparative analysis it is found that the AFNCS has achieved better results than Ferdouse et al. [22]. Adaptation capability of the system to any input noise situation as well as the impacts of step size, number of filter coefficients, number of samples and input noise level on the performance of the system are thoroughly studied considering a speech signal as useful signal. It is evident that individually each of these parameters has an optimum value at which the adaptive noise canceller showed best performance. AFNCS accomplishes very less output signal error as compared to the conventional baselines. Also, incorporation of less hidden layers resulted in negligible computational delay along with effective utilization of memory.

\section{REFERENCES}

[1] Adnan Quadri, Mohsen Riahi Manesh, and Naima Kaabouch, "Denoising Signals in Cognitive Radio Systems: An Evolutionary Algorithm Based Adaptive Filter," IEEE Annual Ubiquitous Computing, Electronics \& Mobile Communication Conference, pp. 1-6, March 18, 2016, DOI: 10.1109/UEMCON.2016.7777854

[2] Mohsen Riah iManesh, Adnan Quadri, Sriram Subramanian, and Naima Kaabouch, "An Optimized SNR Estimation Technique Using Particle Swarm Optimization Algorithm," IEEE Consumer Communications and Networking Conference, pp. 1-7, 2017, DOI: 10.1109/CCWC.2017.7868387

[3] Adnan Quadri, Mohsen RiahiManesh, and Naima Kaabouch, "Performance Comparison of Evolutionary Algorithms for Noise Cancellation in Cognitive Radio Systems," IEEE Consumer Communications and Networking Conference, pp.1-6, 2017, DOI: 10.1109/CCWC.2017.7868388

[4] R. Tandra and A. Sahai, "Fundamental limits on detection in low SNR under noise uncertainty," Proceedings of the International Conference on Wireless Networks, Communications and Mobile Computing, vol. 1, pp. 464-469, Maui, Hawaii, USA, June 2005, DOI: 10.1109/WIRLES.2005.1549453

[5] Y. Zeng, Y. Liang, A. Hoang, and R. Zhang, "A Review on Spectrum Sensing for Cognitive Radio: Challenges and Solutions," EURASIP Journal on Advances in Signal Processing, vol. 2010, pp. 1-16, 2010, DOI https://doi.org/10.1155/2010/381465

[6] M. Z. Alom and H. J. Lee, "Performance Analysis of Beamforming Algorithm for Noise Cancellation with Respect to the Arrival Angles of Interference Signal," International Conference on Cyber-Enabled Distributed Computing and Knowledge Discovery, Huangshan, pp. 396-399, 2010, DOI: 10.1109/CyberC.2010.78

[7] Akingbade, Kayode F., and Isiaka A. Alimi. "Separation of Digital Audio Signals using LeastMean Square (LMS) Adaptive Algorithm." International Journal of Electrical and Computer Engineering,4.4 (2014): 557, DOI http://dx.doi.org/10.11591/ijece.v4i4.6219

[8] Mohammed, Jafar Ramadhan. "Low complexity adaptive noise canceller for mobile phones based remote health monitoring." International Journal of Electrical and Computer Engineering,4.3 (2014): 422, DOI:http://dx.doi.org/10.11591/ijece.v4i3.5534

[9] J. Yoon, H. Zhang, S. Banerjee and S. Rangarajan, "Video Multicast With Joint Resource Allocation and Adaptive Modulation and Coding in 4G Networks," IEEE/ACM Transactions on Networking, vol. 22, no. 5, pp. 1531-1544, Oct. 2014, DOI: 10.1109/TNET.2013.2279887

[10] S. K. Roy and C. F. Rodrigues, "Echo Canceller Using Error Back Propagation Algorithm," 2014 International Conference on Soft Computing and Machine Intelligence, New Delhi, pp. 98-101,2014, DOI: 10.1109/ISCMI.2014.33

[11] M. A. Ruder, A. M. Lehmann, R. Schober and W. H. Gerstacker, "Single Antenna Interference Cancellation for GSM/VAMOS/EDGE Using \$L_\{p\}\$-Norm Detection and Decoding," in IEEE Transactions on Wireless Communications, vol. 14, no. 5, pp. 2413-2425, May 2015, DOI: 10.1109/TWC.2014.2386861

[12] Y. Zhang et al., "A Multi-Frequency Multi-Standard Wideband Fractional-\$ \{ N\}\$ PLL With Adaptive Phase-Noise Cancellation for Low-Power Short-Range Standards," IEEE Transactions on Microwave Theory and Techniques, vol. 64, no. 4, pp. 1133-1142, April 2016, DOI: 10.1109/TMTT.2016.2536022

[13] J. W. Kelly, D. P. Siewiorek, A. Smailagic and W. Wang, "An Adaptive Filter for the Removal of Drifting Sinusoidal Noise Without a Reference," in IEEE Journal of Biomedical and Health Informatics, vol. 20, no. 1, pp. 213-221, Jan. 2016, DOI: 10.1109/JBHI.2014.2375318

[14] R. R. Chilipi, N. Al Sayari, A. R. Beig and K. Al Hosani, "A Multitasking Control Algorithm for Grid-Connected Inverters in Distributed Generation Applications Using Adaptive Noise Cancellation Filters," in IEEE Transactions on Energy Conversion, vol. 31, no. 2, pp. 714-727, June 2016, DOI: 10.1109/TEC.2015.2510662

[15] J. Arenas-Garcia, L. A. Azpicueta-Ruiz, M. T. M. Silva, V. H. Nascimento and A. H. Sayed, "Combinations of Adaptive Filters: Performance and convergence properties," in IEEE Signal Processing Magazine, vol. 33, no. 1, pp. 120-140, Jan. 2016, DOI: 10.1109/MSP.2015.2481746 
[16] Dixit, Shubhra, and Deepak Nagaria. "LMS Adaptive Filters for Noise Cancellation ;A review."International Journal of Electrical and Computer Engineering,7.5 (2017): 2520, DOI: http://doi.org/10.11591/ijece.v7i5.pp2520-2529

[17] Z. Zheng, Z. Liu, H. Zhao, Y. Yu and L. Lu, "Robust Set-Membership Normalized Subband Adaptive Filtering Algorithms and Their Application to Acoustic Echo Cancellation," in IEEE Transactions on Circuits and Systems I: Regular Papers, vol. 64, no. 8, pp. 2098-2111, Aug. 2017, DOI: 10.1109/TCSI.2017.2685679

[18] S. Zhang, W. X. Zheng, J. Zhang and H. Han, "A Family of Robust M-Shaped Error Weighted Least Mean Square Algorithms: Performance Analysis and Echo Cancellation Application," in IEEE Access, vol. 5, pp. 14716-14727, 2017, DOI: 10.1109/ACCESS.2017.2722464

[19] M. A. Ahmed and C. C. Tsimenidis, "Tight Upper Bound Performance of Full-Duplex MIMO-BICM-IDD Systems in the Presence of Residual Self-Interference," in IEEE Transactions on Wireless Communications, vol. 17, no. 1, pp. 520-532, Jan. 2018, DOI: 10.1109/TWC.2017.2768027

[20] E. C. Mengüç, "Novel quaternion-valued least-mean kurtosis adaptive filtering algorithm based on the GHR calculus," in IET Signal Processing, vol. 12, no. 4, pp. 487-495, 2018, DOI: 10.1049/iet-spr.2017.0340

[21] Kumar, AM Prasanna, and K. Ramesha. "Adaptive Filter Algorithms Based Noise Cancellation Using Neural Network in Mobile Applications,"International Conference on Intelligent Computing and Applications. Springer, Singapore, 2018, DoI https://doi.org/10.1007/978-981-10-5520-1_8

[22] Ferdouse L, Akhter N, Nipa TH, Jaigirdar FT. "Simulation and performance analysis of adaptive filtering algorithms in noise cancellation," arXiv preprint arXiv,1104.1962. Apr 2011., DOI arXiv:1104.1962v1

[23] Farhana Afroz, Asadul Huq, F.Ahmed and Kumbesan Sandrasegaran, " Performance Analysis of Adaptive Noise Canceller Employing NLMS Algorithm," International Journal of Wireless \& Mobile Networks (IJWMN) Vol. 7, No. 2, April 2015, DOI: 10.5121/ijwmn.2015.7204

\section{BIOGRAPHY OF AUTHORS}

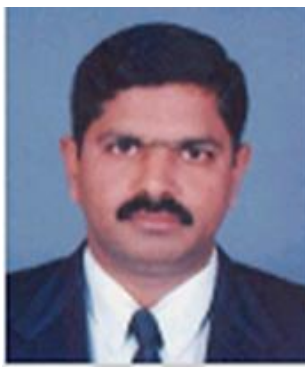

Dr.A.M.Prasanna Kumar obtained B.E degree during 1989 in Electronics \& Communication Engineering from Mysore University and M.E degree during 1993 in Electronics from Gulbarga University \& earned Ph.D in Electronics \& Communication Engineering from Visvesvaraya Technological University, Belagavi. He has 6 research publication in International Journals and Conference Proceedings. He has guided PG \& UG Projects. He is currently working as Professor in E\&CE Department of ACS College of Engineering, Bangalore. His research interests include Adaptive Algorithms, Image Processing,VLSI, Neural Networks and Mobile Applications. He is a life member of Indian Society for Technical Education, New Delhi. He is a Fellow member of IETE (FIE).

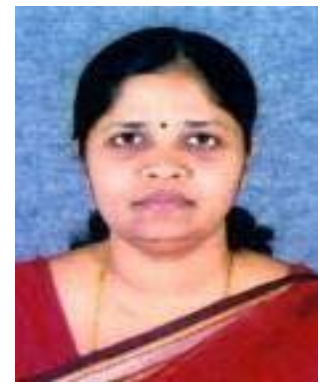

Dr.Vijaya S.M obtained B.E during 1990 in Electronics and Communication Engineering from Gulbarga University \& M.Tech during 2005 in Applied Electronics \& earned Ph.D in Electronics \& Communication Engineering from Visvesvaraya Technological University, Belagavi. Presently she is working as Associate Professor in Electronics \& Communication Engineering Department of RajaRajeswari College of Engineering, Bangalore. She has altogether 18 years of Teaching experience. She has published 6 International journal papers \& 8 Conference papers. She has guided PG \& UG Projects. Her areas of interest are Image Processing and VLSI. She is a Life member of ISTE \& IETE. 